This is a postprint version of the following published document:

Zheng, $X$., et al. Dynamics of necking and fracture in ductile porous materials, In: Journal of applied Mechanics, 87(4), 041005, Apr 2020, 10 $p p$.

DOI: https://doi.org/10.1115/1.4045841

(C) 2020 by ASME. 


\title{
Dynamics of necking and fracture in ductile porous materials
}

\author{
Xinzhu Zheng \\ Department of Materials Science and Engineering \\ Texas A\&M University, College Station, Texas, USA \\ Email: xinzhu_zheng@tamu.edu \\ Komi E. N'souglo \\ Department of Continuum Mechanics and Structural Analysis \\ University Carlos III of Madrid, Madrid, Spain \\ Email: knsouglo@ing.uc3m.es \\ Jose A. Rodriguez-Martinez \\ Department of Continuum Mechanics and Structural Analysis \\ University Carlos III of Madrid, Madrid, Spain \\ Email: jarmarti@ing.uc3m.es \\ Ankit Srivastava * \\ Department of Materials Science and Engineering \\ Texas A\&M University, College Station, Texas, USA \\ Email: ankit.sri@tamu.edu
}

The onset of necking in dynamically expanding ductile rings is delayed due to the stabilizing effect of inertia, and with increasing expansion velocity, both the number of necks incepted and the number of fragments, increase. In general, neck retardation is expected to delay fragmentation as necking is often the precursor to fracture. However, in porous ductile materials it is possible that fracture can occur without significant necking. Thus, the objective of this work is to unravel the complex interaction of initial porosity and inertia on the onset of necking and fracture. To this end, we have carried out a series of finite element calculations of unit cells with sinusoidal geometric perturbations and varying levels of initial porosity under a wide range of dynamic loading conditions. In the calculations, the material is modeled using a constitutive framework that includes many of the hardening and softening mechanisms that are characteristics of ductile metallic materials, such as strain hardening, strain rate hardening, thermal softening and damage-induced softening. The contribution of the inertia effect to the loading process is evaluated through a dimensionless parameter that com-

${ }^{*}$ Corresponding author bines the effects of loading rate, material properties and unit cell size. Our results show that low initial porosity levels favor necking before fracture, and high initial porosity levels favor fracture before necking, especially at high loading rates where inertia effects delay the onset of necking. The finite element results are also compared with the predictions of linear stability analysis of necking instabilities in porous ductile materials.

\section{Introduction}

The analysis of a radially expanding circular ring is a convenient and effective technique to study multiple necking and fragmentation response of metallic materials subjected to dynamic loading conditions [1-18]. The primary advantage of this analysis is that the geometric and loading symmetries nearly eliminate the effects of wave propagation along the circumferential direction of the specimen before plastic flow localizes in the form of multiple necks [19]. In a radially expanding circular ring, both the number of necks incepted and the proportion of necks leading to fragments, increase with increasing expansion velocity $[2-4,10,11,17,18,20,21]$. Furthermore, the stabilizing effects of inertia (and material viscosity to a lesser extent) at high expansion ve- 
locities delay the onset of plastic flow localization (and necking) and gives rise to a post-critical deformation regime $[4,17]$, so that the necks are not incepted at the maximum load (or Considère strain) and are significantly delayed [21]. Intuitively, neck retardation due to the stabilizing effects of inertia will also delay fragmentation as necking is often the precursor to fracture in ductile metallic materials. However, for porous ductile materials it is possible that fracture can occur without significant necking. The objective of this work is to unravel the complex interaction of initial porosity and inertia on the onset of multiple necking and fracture under dynamic loading conditions consistent with expanding rings.

Several authors have carried out finite element calculations of multiple necking in dynamically expanding circular rings, or in long cylindrical bars with initial conditions consistent with the expanding ring [13,17,20-22]. For the initial and boundary conditions applied in these finite element calculations, in the absence of any perturbation, the ring expands or the bar stretches uniformly during loading, according to the fundamental solution of the problem, and localization never occurs [21]. In order to break the symmetry of the problem and trigger localization a perturbation such as geometric or material heterogeneity is readily introduced [20-22]. Note, that some commercial finite element codes such as ABAQUS/Explicit [23], introduce numerical perturbations (likely due to round-off errors) that are sufficient to break the symmetry and trigger localization $[13,17,20]$. The amplitude and distribution of the geometrical or material heterogeneities have been shown to affect the multiple necking pattern (i.e. number, location and growth of the necks) at low loading rates $[21,22]$. However, at high loading rates where inertia effects dominate, the effect of geometrical or material heterogeneities on multiple necking pattern becomes less significant $[21,22]$. Furthermore, it has also been shown that the variations in material properties that greatly affect the multiple necking pattern and fragmentation at relatively low loading rates [13] becomes less significant at high loading rates [17]. The variation in the average neck spacing i.e. the perimeter of the ring or the length of the bar divided by the number of necks incepted, as a function of the imposed loading rate obtained from the finite element calculations [20,21] have shown to be in good agreement with the experimental results [2].

Another approach to analyzing multiple necking and fragmentation in dynamically expanding circular rings is through unit cell finite element calculations introduced in Xue et al. [24]. The unit cell model is based on the idea that a radially expanding circular ring with periodic perturbations is simply a collection of unit cells with sinusoidal perturbations. By utilizing these unit cells of different sizes (defined in terms of length to diameter ratios) and initial conditions consistent with the expanding ring, Rodríguez-Martínez et al. [17] modeled the dependence of the average neck spacing on material properties and imposed loading rates. In these calculations, material was assumed to be plastically incompressible and follow power law strain rate hardening. Material failure or fracture was not considered in this work and the necking strain, i.e. the strain at the ends of the unit cells at which the plastic flow localizes in the center of the cell was measured. These calculations show that the necking strain first decreases with increasing cell size and then starts to increase for cell sizes greater than a critical value. So that the minimum necking strain corresponds to an intermediate unit cell size. In ref. [17], the unit cell size with the minimum necking strain, the critical cell size, was obtained for a range of imposed loading rates, and for materials with different densities and strain rate sensitivities. These results were compared with the average neck spacing obtained from the finite element calculations of radially expanding circular rings and a very good quantitative agreement between the two approaches was noted. This suggests that, for a fixed loading rate and material properties, the critical cell size for which the deformation energy required to trigger a neck is minimum, represents the average neck spacing in the multiple necking pattern.

The unit cell finite element calculations introduced in ref. [24] and utilized in ref. [17] provide a simple computational tool to model the effect of initial porosity on multiple necking and fracture under dynamic loading conditions. To this end, we carry out three-dimensional unit cell finite element calculations for a wide range of the aspect ratio of the cells, and amplitudes of the initial sinusoidal geometric perturbation. The material is modeled using an elastic-viscoplastic constitutive relation for a progressively cavitating ductile solid. The constitutive relation also includes strain hardening, strain rate hardening and thermal softening. Fracture is assumed to occur when a critical level of porosity is reached as in ref. [20]. We note that previous works on multiple necking and fragmentation assumed the material to be plastically incompressible with few notable exceptions such as refs. [7, 20,21] which considered porous plasticity, but in these works the interplay between porosity and inertia was not explored. Here, the calculations are carried out for different levels of the initial porosity in the material. The contribution of the inertia effects to the loading process is evaluated through a dimensionless parameter that combines the effects of loading rate, material properties and unit cell size. The unit cell finite element calculations are carried out for a range of imposed uniaxial velocities giving a wide variation in the value of the dimensionless inertia parameter. The finite element results are also compared with the predictions of linear stability analysis of necking instabilities in porous ductile materials developed in ref. [21]. The linear stability analysis, despite several simplifying assumptions, provides a quick understanding of the fundamental problem and enables us to: (i) obtain the 
fundamental dimensionless parameters that govern the necking instabilities; (ii) rationalize the stabilizing effects of stress multiaxiality and inertia; and (ii) provide additional verification of the effect of porosity on necking instabilities in ductile porous materials subjected to dynamic loading conditions.

\section{Constitutive framework}

The constitutive framework used is the modified Gurson elastic-viscoplastic constitutive relation for a progressively cavitating solid $[25,26]$ with the flow potential having the form

$$
\Phi=\frac{\sigma_{e}^{2}}{\bar{\sigma}^{2}}+2 q_{1} f^{*} \cosh \left(\frac{3 q_{2} \sigma_{h}}{2 \bar{\sigma}}\right)-1-\left(q_{1} f^{*}\right)^{2} \leq 0
$$

where $q_{1}=1.25, q_{2}=1$ are the material parameters introduced in refs. [27,28], $\bar{\sigma}$ is the matrix flow strength, and

$$
\sigma_{e}=\sqrt{\frac{3}{2} \boldsymbol{\sigma}^{\prime}: \boldsymbol{\sigma}^{\prime}}, \quad \sigma_{h}=\frac{1}{3} \boldsymbol{\sigma}: \mathbf{1}, \quad \boldsymbol{\sigma}^{\prime}=\boldsymbol{\sigma}-\sigma_{h} \mathbf{1}
$$

with $\boldsymbol{\sigma}$ being the Cauchy stress tensor and $\mathbf{1}$ being the unit second-order tensor. by

Following, ref. [26] the function $f^{*}$ in Eq. (1) is given

$$
f^{*}=\left\{\begin{array}{lll}
f & \text { if } & f<f_{c} \\
f_{c}+\frac{\left(1 / q_{1}-f_{c}\right)\left(f-f_{c}\right)}{\left(f_{f}-f_{c}\right)} & \text { if } & f \geq f_{c}
\end{array}\right.
$$

where $f$ is the void volume fraction, $f_{c}$ is the critical void volume fraction to void coalescence and $f_{f}$ is the void volume fraction at failure. The values of $f_{c}=0.12$ and $f_{f}=0.25$ are used in the calculations.

The rate of deformation tensor is written as the sum of an elastic part, $\mathbf{d}^{e}=\mathbf{L}^{-1}: \hat{\boldsymbol{\sigma}}$, a plastic part, $\mathbf{d}^{p}$, and a part due to thermal straining, $\mathbf{d}^{\Theta}=\alpha \dot{\Theta} \mathbf{1}$, so that

$$
\mathbf{d}=\mathbf{L}^{-1}: \hat{\boldsymbol{\sigma}}+\alpha \dot{\Theta} \mathbf{1}+\mathbf{d}^{p}
$$

Here, $\hat{\boldsymbol{\sigma}}$ is the Jaumann rate of Cauchy stress, $\Theta$ is the temperature, $\alpha=1 \times 10^{-5} \mathrm{~K}^{-1}$ is the thermal expansion coefficient and $\mathbf{L}=\lambda \mathbf{1} \otimes \mathbf{1}+2 \mu \mathbf{I}$ is the tensor of isotropic elastic moduli, where $\lambda=40.38 \mathrm{GPa}$ and $\mu=26.92 \mathrm{GPa}$ are the Lamé constants, and $\mathbf{I}$ is the fourth-order unit tensor. The plastic part of the rate of deformation tensor, $\mathbf{d}^{p}$, as formulated in ref. [29], is written as

$$
\mathbf{d}^{p}=\left[\frac{(1-f) \bar{\sigma} \dot{\bar{\varepsilon}}^{p}}{\boldsymbol{\sigma}: \frac{\partial \Phi}{\partial \boldsymbol{\sigma}}}\right] \frac{\partial \Phi}{\partial \boldsymbol{\sigma}}
$$

The effective plastic strain rate in the matrix material, $\dot{\bar{\varepsilon}} p$, is given by

$$
\dot{\bar{\varepsilon}}^{p}=\dot{\varepsilon}_{0}\left[\frac{\bar{\sigma}}{g\left(\bar{\varepsilon}^{p}, \Theta\right)}\right]^{1 / m}
$$

with

$$
g\left(\bar{\varepsilon}^{p}, \Theta\right)=\sigma_{0} G(\Theta)\left[1+\bar{\varepsilon}^{p} / \varepsilon_{0}\right]^{N}
$$

and

$G(\Theta)=1+b_{G} \exp \left(-c\left[\Theta_{0}-273\right]\right)\left[\exp \left(-c\left[\Theta-\Theta_{0}\right]\right)-1\right]$

where $\bar{\varepsilon}^{p}=\int \dot{\bar{\varepsilon}} p d t$ is the effective plastic strain in the matrix material. In the calculations, the values of initial flow strength of the matrix material $\sigma_{0}=300 \mathrm{MPa}$, strain hardening exponent $N=0.1$, strain rate sensitivity exponent $m=0.01$, reference strain $\varepsilon_{0}=0.0043$, reference strain rate $\dot{\varepsilon}_{0}=10^{3} \mathrm{~s}^{-1}, b_{G}=0.1406$ and $c=0.00793 \mathrm{~K}^{-1}$ are used, and $\Theta_{0}=293 \mathrm{~K}$ is the initial temperature of the material.

The evolution of the void volume fraction is governed by

$$
\dot{f}=(1-f) \mathbf{d}^{p}: \mathbf{1}
$$

where the value of $f$ in the undeformed material, i.e. the value of $f$ at time $t=0$, represents the initial void volume fraction (or porosity), $f_{0}$. For, $f_{0}=0$, the material is fully dense and follows von Mises plasticity.

Adiabatic conditions are assumed so that

$$
\rho c_{p} \frac{\partial \Theta}{\partial t}=\chi \boldsymbol{\sigma}: \mathbf{d}^{p}
$$

with $\rho$ being the current density of the material (the initial density is $\left.\rho_{0}=7600 \mathrm{~kg} / \mathrm{m}^{3}\right), c_{p}=465 \mathrm{~J} / \mathrm{kg} \mathrm{K}$ is the specific heat, and $\chi=0.9$ is the Taylor-Quinney coefficient. 
The values of all the constitutive and material parameters are taken from ref. [21].

\section{Unit cell finite element model}

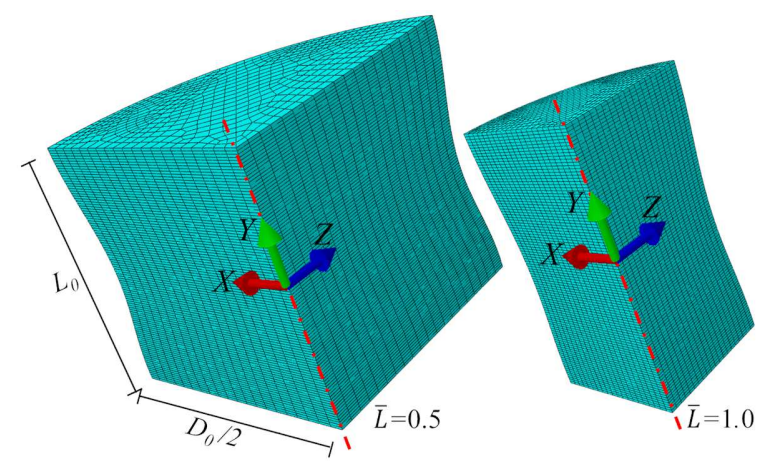

Fig. 1. Finite element mesh of two $1 / 4$ unit cells with normalized cell lengths $\bar{L}=0.5$ and 1 , with a small sinusoidal imperfection of wavelength $L_{0}$, and amplitude $A=0.02$.

We carry out three-dimensional unit cell finite element calculations as in refs. $[17,24]$ using the constitutive framework described in Section 2 to model necking and fracture in porous metallic bars subjected to dynamic loading conditions consistent with expanding ring. The finite element calculations are based on the dynamic principle of virtual work using a finite deformation Lagrangian convected coordinate formulation. Material points are referred to using a Cartesian coordinate system with positions in the reference configuration denoted as $(X, Y, Z)$. The origin of the coordinate system is located at the center of mass of the unit cell. The unit cell modeled is a cylindrical bar with initial length, $L_{0}$, and diameter, $D$, with initial sinusoidal geometric imperfection:

$$
D(Y)=D_{0}\left[1-A\left(1+\cos \left(\frac{2 \pi Y}{L_{0}}\right)\right)\right]
$$

where, $D_{0}$ is the initial unperturbed diameter of the unit cell, and $A$ is the amplitude of the imperfection. The coordinate along the axis of the unit cell, $Y$, in the reference configuration varies from $-L_{0} / 2$ to $L_{0} / 2$. Owing to the symmetry of the cross-section of the cylindrical unit cell, only $1 / 4$ of the specimen is analyzed numerically, Fig. 1. Although, three-dimensional unit cell finite element calculations are carried out in this work, the unit cell in Fig. 1, is axisymmetric about the $Y$ axis and can also be analyzed numerically using a simpler axisymmetric finite element model.

Two representative configurations of the unit cell with normalized cell lengths $\bar{L}=L_{0} / D_{0}=0.5$ and 1 , and with amplitude $A=0.02$, of the sinusoidal imperfection are shown in Fig. 1. The finite element mesh of the unit cell with $\bar{L}=0.5$ consists of 55,200 twenty node brick elements while that of the unit cell with $\bar{L}=1$ consists of 53,800 twenty node brick elements. The initial and boundary conditions in velocity imposed on the unit cell are,

$$
\begin{aligned}
& V_{Y}(X, Y, Z, 0)=V_{0} \times \frac{Y}{L_{0} / 2} \\
& V_{Y}\left(X, \pm L_{0} / 2, Z, t\right)= \pm V_{0}
\end{aligned}
$$

where, $V_{Y}$ is the velocity along the axis of the unit cell, $t$ is the analysis time and $V_{0}$ is the magnitude of the imposed velocity. The unit cell finite element model considered in this work is based on the idea that a radially expanding circular ring with periodic perturbations can be represented as a collection of unit cells (shown in Fig. 1). Note that in a radially expanding ring the geometric and loading symmetries nearly eliminate wave propagation along the circumferential direction. Following this, the initial condition in Eq. (12) is utilized to minimize wave propagation along the axial direction of the unit cell (which correspond to the circumferential direction of the ring). In a radially expanding ring, wave propagation can occur in other directions, assuming this, no initial conditions are utilized along any other direction of the unit cell in this work. Also, in ref. [17], the critical neck size as a function of the imposed strain rate in a plastically incompressible material obtained from full three-dimensional finite element calculations of radially expanding rings were found to be in quantitative agreement with the predictions of the unit cell finite element calculations with initial and boundary conditions same as in Eq. (12). Nonetheless, we carried out limited calculations to check the effect of initializing velocity along the radial direction on the predictions of the unit cell finite element calculations similar to ref. [20]. Our results, that are not presented in this paper for the sake of brevity, show that the effect of the initial velocity along the radial direction has negligible influence on the evolution of plastic strain and porosity. However, the initial velocity along the radial direction does slightly reduce the oscillations in the axial force for unit cells with small wavelengths.

As in refs. [21,30-33], eight point Gaussian integration is used in each twenty-node element for integrating the internal force contributions and twenty-seven point Gaussian integration is used for the element mass matrix. Lumped masses are used so that the mass matrix is diagonal. The discretized equations are integrated using the explicit Newmark $\beta$-method $(\beta=0)$ [34]. The constitutive updating is based on the rate tangent modulus method proposed in ref. [35], while material failure is implemented via the element vanishing technique proposed in ref. [36]. When the value of the void volume fraction $f$ at an integration point reaches $0.9 f_{f}$, the 
value of $f$ is kept fixed so that the material deforms with a very low flow strength. The entire element is taken to vanish when three of the eight integration points in the element have reached this stage.

The unit cell finite element calculations are carried out for nine unit cells with normalized cell length, $\bar{L}$ (Fig. 1), varying from 0.25 to 3 . For all unit cells, the initial length $L_{0}=1 \mathrm{~mm}$ is kept fixed and the initial diameter is varied as, $D_{0}=L_{0} / \bar{L}$. For each unit cell, the calculations are carried out for three amplitudes of the sinusoidal geometric imperfection, $A=0.002,0.005$ and 0.02 , four initial porosity levels, $f_{0}=0,0.01,0.05$ and 0.08 , and a range of imposed velocities, $V_{0}$. The initial porosity levels assumed in our calculations are rather high compared to conventionally processed engineering metals and alloys. However, with the emergence of manufacturing technologies such as additive manufacturing, it is becoming possible to control the initial porosity levels in a material. Our objective here is to unravel the interaction of initial porosity and inertia on the onset of multiple necking and fracture under dynamic loading conditions. This will provide an understanding of the extent to which the initial porosity level in a material can be controlled to engineer multiple necking and fracture pattern under dynamic loading conditions. From the unit cell finite element calculations, strain to failure dictated by necking and/or fracture are measured. Our finite element results presented in Section 5 show that the failure strain dictated by necking first decreases with increasing value of $\bar{L}$ and then starts to increase for values of $\bar{L}$ greater than a critical value. The value of $\bar{L}$ for which the necking strain is minimum is referred to as the critical cell size, $\bar{L}_{c}$. The value of $\bar{L}_{c}$, for which the deformation energy required to trigger failure is minimum, likely represents the average neck spacing in the multiple necking pattern as noted in the Section 1.

\section{Theoretical model}

In this section, we briefly summarize the main features of the state-of-the-art one-dimensional linear stability analysis developed in ref. [21], to model necking instabilities in porous metallic bars subjected to dynamic loading conditions consistent with expanding ring. The theoretical model developed in ref. [21] for the constitutive framework detailed in Section 2 includes both the effects of inertia and multiaxial stress state that develops inside the necked region.

The linear stability analysis technique to study the formation of dynamic necking instabilities $[37,38]$ involves testing the stability of the fundamental solution of the problem $\mathbb{S}_{1}$ at any time $t_{1}$ by introducing into the governing equations a small perturbation of the form

$$
\delta \mathbb{S}(\mathrm{Y}, \mathrm{t})_{\mathrm{t}_{1}}=\delta \mathbb{S}_{1} e^{i \xi \mathrm{Y}+\eta\left(\mathrm{t}-\mathrm{t}_{1}\right)}
$$

where $\delta \mathbb{S}_{1}$ is the perturbation amplitude, $\xi$ is the wavenumber and $\eta$ is the growth rate of the perturbation at time $t_{1}$. The wavenumber is related to the normalized perturbation wavelength as $\bar{L}=\frac{1}{D_{0}} \frac{2 \pi}{\xi}$. The normalized perturbation wavelength is analogous to the normalized unit cell length in the finite element calculations.

The perturbed solution of the problem is given by

$$
\mathbb{S}=\mathbb{S}_{1}+\delta \mathbb{S}
$$

with $|\delta \mathbb{S}| \ll\left|\mathbb{S}_{1}\right|$. By inserting Eq. (14) into the governing equations of the problem, and keeping only the first-order terms in the increments $\delta \mathbb{S}_{1}$, linearized equations are obtained. A non-trivial solution for $\delta \mathbb{S}_{1}$ can only be obtained if the determinant of the system of linearized equations is equal to zero. Application of this condition leads to a fourth-degree polynomial in $\eta$

$$
\begin{aligned}
& B_{4}\left(\mathbb{S}_{1}, \xi\right) \eta^{4}+B_{3}\left(\mathbb{S}_{1}, \xi\right) \eta^{3}+ \\
+ & B_{2}\left(\mathbb{S}_{1}, \xi\right) \eta^{2}+B_{1}\left(\mathbb{S}_{1}, \xi\right) \eta+B_{0}\left(\mathbb{S}_{1}, \xi\right)=0
\end{aligned}
$$

with coefficients $B_{i}\left(\mathbb{S}_{1}, \xi\right)$ that depend on the fundamental solution and the wavenumber. Equation (15) has four roots in $\eta$, two real and two complex conjugates. The requisite for unstable growth of $\delta \mathbb{S}$ is given by $\operatorname{Re}(\eta)>0$ and hence the root that has the greater positive real part, $\eta^{+}$, is considered for the analysis. The stabilizing effect of inertia and multiaxial stress state on small and large wavenumbers, respectively, promotes the growth of intermediate modes [8,38-41]. To track the history of the growth rate of all the growing modes during the post-homogeneous deformation process (i.e. for strains greater than the Considère strain), we use the cumulative instability index $I=\int_{0}^{t} \eta^{+} d t[21,38,42,43]$. The index $I$ accumulates the growth rate of all the growing modes during the loading process, i.e. we introduce the perturbation at different times and sum the growth rate obtained for each loading time. At a given loading time $t$, i.e. at a given strain $\varepsilon$, the mode that grows the fastest, i.e. the mode with greatest value of $I$, is referred to as critical wavenumber or critical perturbation mode $\xi_{c}$. Likewise, the greatest value of $I$ is referred to as critical cumulative instability index, $I_{c}$. The critical wavenumber enables us to calculate the critical normalized perturbation wavelength, $\bar{L}_{c}=\frac{1}{D_{0}} \frac{2 \pi}{\xi_{c}}$, which is analogous to the critical unit cell size, $\bar{L}_{c}$, obtained from the finite element calculations.

\section{$5 \quad$ Results and discussions}

This section is divided into two sub-sections. Section 5.1 presents the key results and discussion of the unit cell finite element calculations and Section 5.2 com- 
pares the unit cell finite element results with the predictions of the linear stability analysis. Both, the unit cell finite element calculations and the linear stability analyses are carried out for a range of imposed velocities, $V_{0}$, giving a variation in the value of the dimensionless inertia parameter, $\Pi$, defined as

$$
\Pi=\sqrt{\left(V_{0} \frac{D_{0}}{L_{0}}\right)^{2} \frac{\rho_{0}}{\sigma_{0}}}
$$

in the range of 0.05 to 0.3 . The parameter $\Pi$ is the inertial resistance to motion, and allows for the quantification of the dynamic effects $[11,17,21,44]$.

\subsection{Key finite element results}

The evolution of the effective plastic strain, $\bar{\varepsilon}_{m i d}^{p}$, and the porosity, $f$, in the mid-section of two unit cells, $\bar{L}=1.0$ and 0.5 , with the effective plastic strain at the end of the unit cells, $\bar{\varepsilon}_{\text {end }}^{p}$, subjected to $\Pi=0.1$ are shown in Fig. 2. For both the unit cells, the values of $A=0.002$ and $f_{0}=0.01$. As shown in the figure, at the early stages of deformation the values of $\bar{\varepsilon}_{m i d}^{p}$ evolve linearly with $\bar{\varepsilon}_{e n d}^{p}$, Fig. 2(a), with limited increase in the value of $f$, Fig. 2(b). However, with continued deformation, the plastic flow localizes in the mid-section of the unit cells and the value of $\bar{\varepsilon}_{m i d}^{p}$ increases asymptotically while the value of $\bar{\varepsilon}_{\text {end }}^{p}$ remains nearly constant. The value of $\bar{\varepsilon}_{e n d}^{p}$ at which $d \bar{\varepsilon}_{m i d}^{p} / d \bar{\varepsilon}_{e n d}^{p} \rightarrow \infty$ is defined here as the effective plastic strain at the onset of necking. Following the onset of necking, the value of $f$ also increases asymptotically. The onset of necking and the asymptotic increase in the value of $f$ occur at a smaller value of $\bar{\varepsilon}_{\text {end }}^{p}$ in the unit cell with $\bar{L}=1.0$ than in the unit cell with $\bar{L}=0.5$. Furthermore, in the unit cell with $\bar{L}=1.0$, the value of $\bar{\varepsilon}_{e n d}^{p}$ at the onset of necking is smaller in the center of the mid-section of the unit cell compared to the surface of the mid-section of the unit cell. This is because in the unit cell with $\bar{L}=1.0$ plastic flow first localizes in the center and subsequently propagates towards the surface of the mid-section of the unit cell. On the contrary, in the unit cell with $\bar{L}=0.5$ plastic flow first localizes at the surface and subsequently propagates towards the center of the mid-section of the unit cell. Similarly, the onset of the asymptotic growth in the value of $f$ first occurs in the center of the mid-section of the unit cell with $\bar{L}=1.0$, while the onset of the asymptotic growth in the value of $f$ first occurs at the surface of the mid-section of the unit cell with $\bar{L}=0.5$. Here, we identify onset of fracture as the smallest value of $\bar{\varepsilon}_{\text {end }}^{p}$ at which $f>f_{c}$.

In Figs. 2(a) and (b), the value of $\bar{\varepsilon}_{\text {end }}^{p}$ at which necking initiates in the center of the mid-section of the unit cell is marked with open symbols while the closed symbols correspond to the value of $\bar{\varepsilon}_{e n d}^{p}$ at which necking initiates at the surface of the mid-section of the unit cell. Also, in Figs. 2(a) and (b), the value of $\bar{\varepsilon}_{e n d}^{p}$ at which fracture initiates in the center of the mid-section of the unit cell is marked with encircled open symbols while the encircled closed symbols correspond to the value of $\bar{\varepsilon}_{\text {end }}^{p}$ at which fracture initiates at the surface of the mid-section of the unit cell. The variation in the location of the initiation of plastic flow localization for the two unit cells in Fig. 2, is further illustrated via contour plots of effective plastic strain, $\bar{\varepsilon}^{p}$, in Fig. 3. As seen in the figure, in the unit cell with $\bar{L}=1.0$, Fig. 3(a), the value of $\bar{\varepsilon}^{p}$ is maximum in the center of the mid-section of the unit cell whereas in the unit cell with $\bar{L}=0.5$, Fig. 3(b), the value of $\bar{\varepsilon}^{p}$ is maximum at the surface of the mid-section of the unit cell.

For the two cases shown in Fig. 2, the onset of necking precedes the onset of fracture. We now present two examples where the onset of fracture precedes necking for the same imposed inertia parameter $\Pi=0.1$. Fig. 4 shows the evolution of $\bar{\varepsilon}_{m i d}^{p}$ and $f$ with $\bar{\varepsilon}_{\text {end }}^{p}$ in two unit cells. For one unit cell, $\bar{L}=3.0$ and $f_{0}=0.08$, and for the second unit cell, $\bar{L}=0.25$ and $f_{0}=0.05$, while the value of $A=0.002$ is same for both the unit cells. As shown in Fig. 4(a), the value of $\bar{\varepsilon}_{\text {mid }}^{p}$ evolves gradually with the value of $\bar{\varepsilon}_{\text {end }}^{p}$ in both the unit cells and the fracture criteria is met i.e. $f>f_{c}$, see Fig. 4(b), before the necking criteria i.e. $d \bar{\varepsilon}_{m i d}^{p} / d \bar{\varepsilon}_{\text {end }}^{p} \rightarrow \infty$. In the unit cell with $\bar{L}=3.0$ and $f_{0}=0.08$, the value of $\bar{\varepsilon}_{\text {end }}^{p}$ at the onset of fracture is slightly smaller in the center of the mid-section of the unit cell compared to the surface. So that, in this unit cell fracture initiates in the center and subsequently propagates towards the surface. On the contrary, in the unit cell with $\bar{L}=0.25$ and $f_{0}=0.05$, fracture initiates at the surface and subsequently propagates towards the center. The variation in the location of fracture initiation for the two unit cells in Fig. 4, is further illustrated via contour plots of $f$, in Fig. 5. As seen in the figure, in the unit cell with $\bar{L}=3.0$ and $f_{0}=0.08$, Fig. $5(\mathrm{a})$, the value of $f$ is maximum in the center of the mid-section, whereas in the unit cell with $\bar{L}=0.25$ and $f_{0}=0.05$, Fig. $5(\mathrm{~b})$, the value of $f$ is maximum at the surface of the mid-section.

We now analyze the variation of the effective plastic strain at the end of the unit cell at failure, $\left(\bar{\varepsilon}_{e n d}^{p}\right)_{f}$, dictated either by the onset of localization or fracture in the center or at the surface of the mid-section of the unit cell (whichever occurs first), as a function of the normalized unit cell length, $\bar{L}$. The variation of $\left(\bar{\varepsilon}_{\text {end }}^{p}\right)_{f}$ with $\bar{L}$ for four initial porosity levels, $f_{0}$, and for each $f_{0}$, for three values of imposed $\Pi$ are shown in Fig. 6 . In Fig. 6 , the values of $\left(\bar{\varepsilon}_{\text {end }}^{p}\right)_{f}$ set by the onset of localization in the center of the mid-section of the unit cell are marked by the open symbols while the closed symbols mark the onset of localization at the surface of the mid-section of the unit cell. Similarly, the values of $\left(\bar{\varepsilon}_{\text {end }}^{p}\right)_{f}$ set by the onset of fracture in the center of the mid-section of the unit cell are marked by the encircled open symbols while the encircled closed symbols mark 


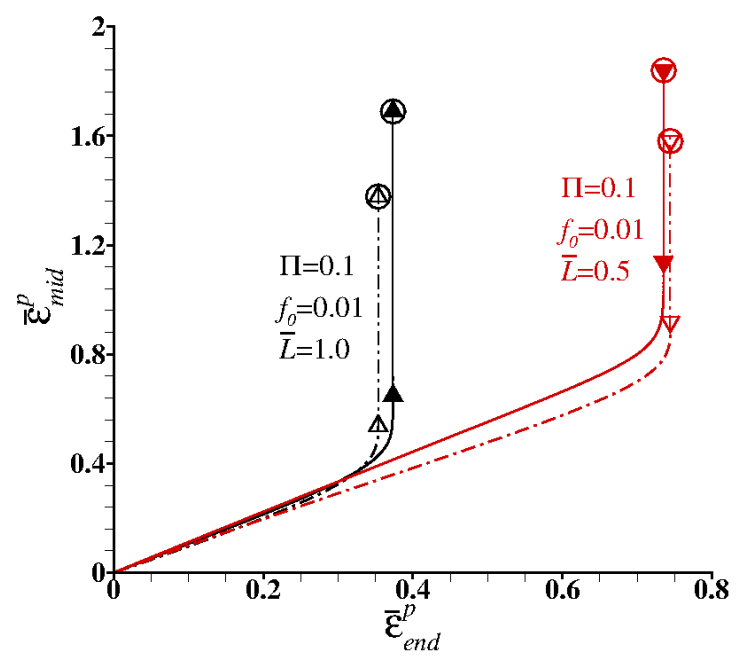

(a)

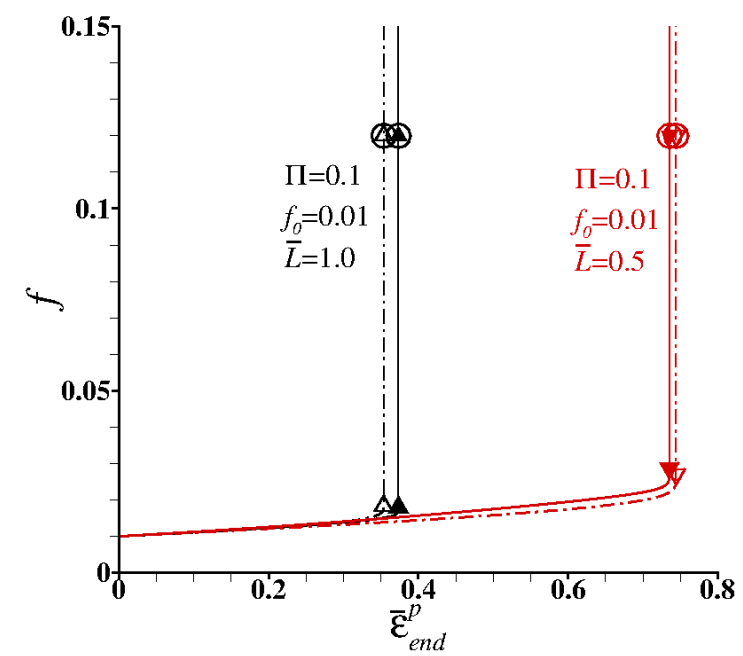

(b)

Fig. 2. Evolution of the (a) effective plastic strain, $\bar{\varepsilon}_{\text {mid }}^{p}$, and (b) porosity, $f$ in the mid-section of the unit cell as a function of the effective plastic strain at the end of the unit cell, $\bar{\varepsilon}_{\text {end }}^{p}$ for two unit cells with normalized cell lengths $\bar{L}=1.0$ and 0.5 , and initial porosity $f_{0}=0.01$. For both the unit cells the amplitude of the imperfection $A=0.002$, and the imposed inertia parameter $\Pi=0.1$. The dash-dot lines show the evolution of $\bar{\varepsilon}_{m i d}^{p}$ and $f$ as a function of $\bar{\varepsilon}_{e n d}^{p}$ in the center while the solid lines show the same at the free surface of the mid-section of the unit cell.

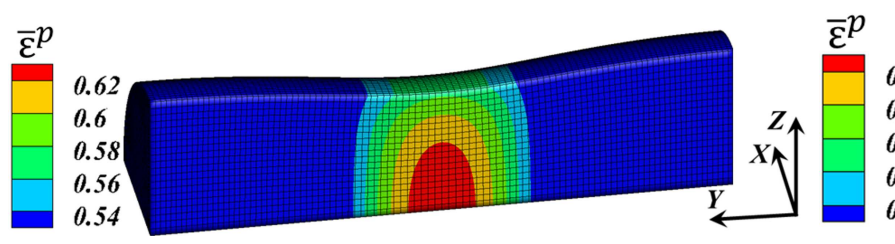

(a)

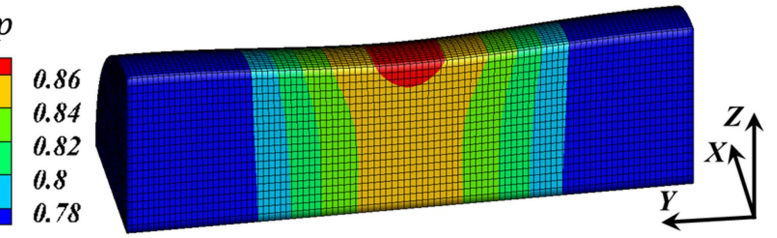

(b)

Fig. 3. The distribution of effective plastic strain, $\bar{\varepsilon}^{p}$, in two unit cells: (a) normalized cell length $\bar{L}=1.0$, initial porosity $f_{0}=0.01$, and deformed to a nominal strain of $\approx 0.61$ with an imposed inertia parameter $\Pi=0.1$, and (b) $\bar{L}=0.5, f_{0}=0.01$ and deformed to a nominal strain of $\approx 1.23$ with $\Pi=0.1$. The amplitude of the imperfection $A=0.002$ for both the unit cells.

the onset of fracture at the surface of the mid-section of the unit cell.

The variation of $\left(\bar{\varepsilon}_{\text {end }}^{p}\right)_{f}$ with $\bar{L}$ for fully dense unit cells i.e. $f_{0}=0$ for three values of imposed $\Pi$ are shown in Fig. 6(a). Since void nucleation is ignored in our calculations, there is no porosity induced fracture in the absence of initial porosity and the values of $\left(\bar{\varepsilon}_{e n d}^{p}\right)_{f}$ are solely dictated by the onset of localization. As shown in Fig. 6(a), for a fixed value of $\Pi$, the value of $\left(\bar{\varepsilon}_{\text {end }}^{p}\right)_{f}$ first decreases with increasing $\bar{L}$, and then starts to increase for values of $\bar{L}$ greater than a critical value. The value of $\bar{L}$ for which $\left(\bar{\varepsilon}_{\text {end }}^{p}\right)_{f}$ is minimum (i.e. the value of $\bar{L}$ for which $\left.d\left(\bar{\varepsilon}_{e n d}^{p}\right)_{f} / d \bar{L}=0\right)$ is the critical cell size, $\bar{L}_{c}$. The greater values of $\left(\bar{\varepsilon}_{e n d}^{p}\right)_{f}$, for small and large normalized cell lengths, are due to the stabilizing effects of stress multiaxiality and inertia, respectively $[8,39]$. For large normalized cell lengths, an increase in the value of imposed $\Pi$ results in a significant increase in the value of $\left(\bar{\varepsilon}_{\text {end }}^{p}\right)_{f}$. This is because the stabilizing effect of inertia increases with increasing value of $\bar{L}$. In contrast, for small normalized cell lengths, there is a very little or no effect of the value of $\Pi$ on $\left(\bar{\varepsilon}_{\text {end }}^{p}\right)_{f}$, because necking and fracture for small normalized cell lengths are controlled by stress multiaxiality. For smaller values of $\bar{L}$, for example $\bar{L}=0.25$ and 0.5 , localization initiates at the surface while for greater values of $\bar{L}$ localization initiates in the center of the mid-section of the unit cells. Due to the stabilizing effects of inertia, the value of $\bar{L}_{c}$ decreases with increasing value of $\Pi$, while the value of $\left(\bar{\varepsilon}_{\text {end }}^{p}\right)_{f}$ corresponding to $\bar{L}_{c}$ increases. For example, the values of $\bar{L}_{c}$ for $\Pi=0.05$ and 0.3 are $\approx 2.5$ and 1.25 , respectively, while the values of $\left(\bar{\varepsilon}_{e n d}^{p}\right)_{f}$ corresponding to $\bar{L}_{c}$ for $\Pi=0.05$ and 0.3 are $\approx 0.14$ and 0.5 , respectively. The values of $\bar{L}_{c}$ for $\Pi=0.05$ and 0.3 in Fig. 6 (a) are comparable to the values reported in ref. [17] for unit cell calculations where the material was assumed to follow von Mises plasticity with strain rate hardening.

In Fig. 6(b), we show the variation of $\left(\bar{\varepsilon}_{e n d}^{p}\right)_{f}$ with $\bar{L}$ for unit cells with initial porosity level $f_{0}=0.01$ for three values of imposed $\Pi$. The value of $f_{0}=0.01$ is 


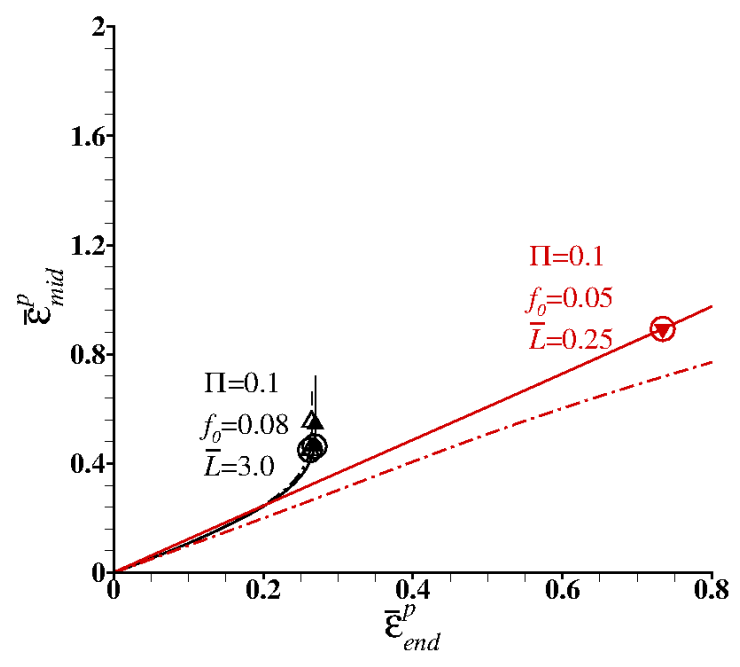

(a)

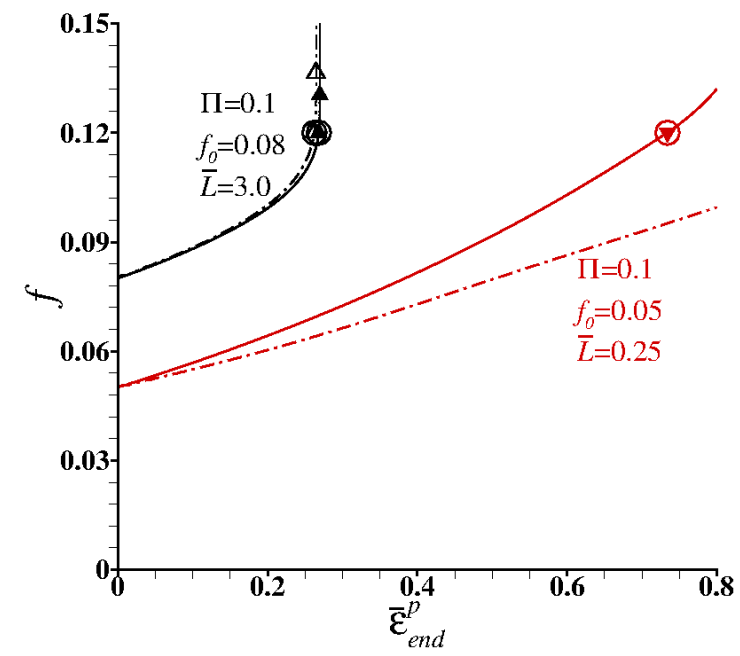

(b)

Fig. 4. Evolution of the (a) effective plastic strain, $\bar{\varepsilon}_{\text {mid }}^{p}$, and (b) porosity, $f$ in the mid-section of the unit cell as a function of the effective plastic strain at the end of the unit cell, $\bar{\varepsilon}_{\text {end }}^{p}$ for two unit cells with normalized cell length $\bar{L}=3$ and initial porosity $f_{0}=0.08$, and $\bar{L}=0.25$ and $f_{0}=0.05$. For both the unit cells the amplitude of the imperfection $A=0.002$, and the imposed inertia parameter $\Pi=0.1$. The dash-dot lines show the evolution of $\bar{\varepsilon}_{m i d}^{p}$ and $f$ as a function of $\bar{\varepsilon}_{\text {end }}^{p}$ in the center while the solid lines show the same at the free surface of the mid-section of the unit cell.

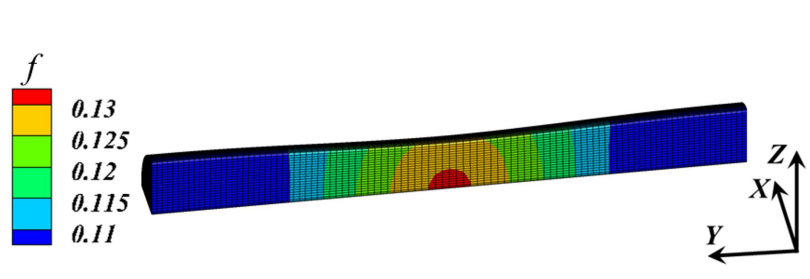

(a)

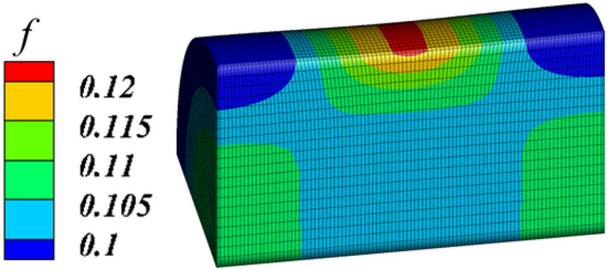

(b)

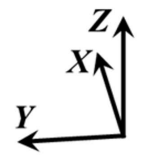

Fig. 5. The distribution of porosity, $f$, in two unit cells: (a) normalized cell length $\bar{L}=3.0$, initial porosity $f_{0}=0.08$, and deformed to a nominal strain of $\approx 0.5$ with an imposed inertia parameter $\Pi=0.1$, and (b) $\bar{L}=0.25, f_{0}=0.05$ and deformed to a nominal strain of $\approx 1.32$ with $\Pi=0.1$. The amplitude of the imperfection $A=0.002$ for both the unit cells.

not sufficient to induce fracture without the onset of localization so that similar to $f_{0}=0$, for $f_{0}=0.01$ as well, the values of $\left(\bar{\varepsilon}_{e n d}^{p}\right)_{f}$ are dictated by the onset of localization. The dependence of the value of $\left(\bar{\varepsilon}_{e n d}^{p}\right)_{f}$ on the value of $\bar{L}$ for all three values of $\Pi$ for unit cells with $f_{0}=0.01$ is roughly the same as for the unit cells with $f_{0}=0$. The values of the $\bar{L}_{c}$ identified from the Figs. 6(a) and (b) as a function of the imposed $\Pi$ are also consistent with the average neck spacing obtained from the analyses of multiple necking in long cylindrical bars modeled with Gurson-type plasticity and the same initial porosity $f_{0}=0.01$, and subjected to initial and loading conditions consistent with the expanding ring [20,21]. In ref. [21], where the same constitutive model and material parameters reported in Section 2 were used, for $\Pi=0.05,0.1$ and 0.3 , the average neck spacing was predicted to be $1.7,1.3$ and 0.9 , respectively. While in ref. [20], where specific material parameters for aluminum and copper were used, for $\Pi=0.05$ the average neck spacing was predicted to be 2.85 for copper and 2.31 for aluminum, and for $\Pi=0.1$ the average neck spacing was predicted to be 2.21 for copper.

The variation of $\left(\bar{\varepsilon}_{e n d}^{p}\right)_{f}$ with $\bar{L}$ for unit cells with initial porosity levels $f_{0}=0.05$ and 0.08 for three values of imposed $\Pi$ are shown in Figs. 6(c) and (d), respectively. For high initial porosity levels, depending on the values of $\bar{L}$ and imposed $\Pi$, the onset of fracture can precede the onset of necking. The value of the $\left(\bar{\varepsilon}_{\text {end }}^{p}\right)_{f}$ for a fixed $\bar{L}$ and imposed $\Pi$, when dictated by the onset of fracture (for high initial porosity levels) is always less than the value of $\left(\bar{\varepsilon}_{e n d}^{p}\right)_{f}$ when dictated by the onset of necking (for low initial porosity levels). As shown in Figs. 6(c) and (d), for unit cells with $f_{0}=0.05$ and 0.08 , fracture precedes necking for smaller values of $\bar{L}$ for all three imposed values of $\Pi$, while for greater values of $\bar{L}$, the propensity for fracture preceding necking increases with increasing value of $f_{0}$ and $\Pi$. For smaller value of $\bar{L}$, when fracture precedes necking, the value of 


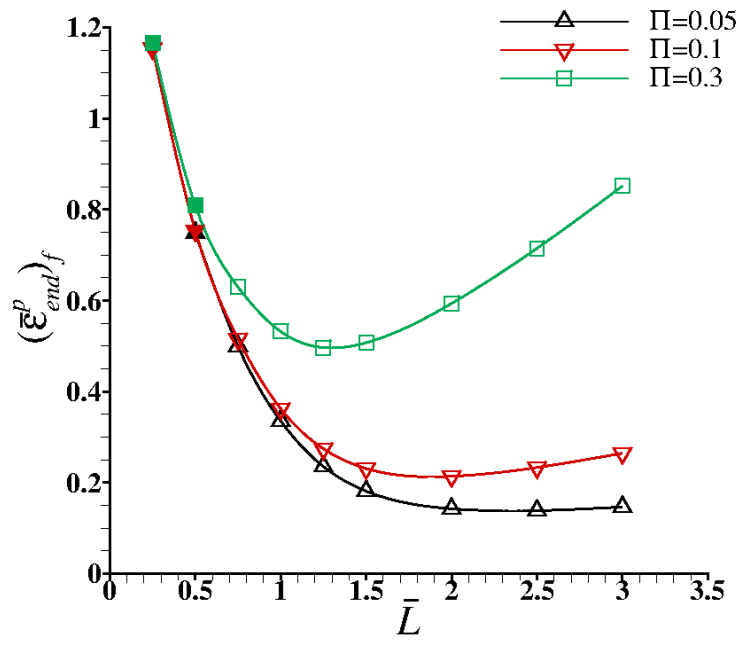

(a)

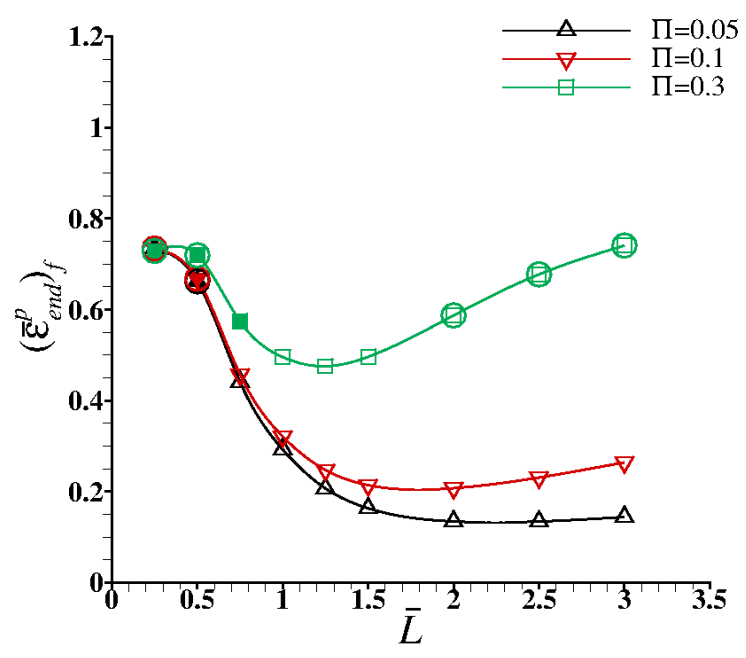

(c)

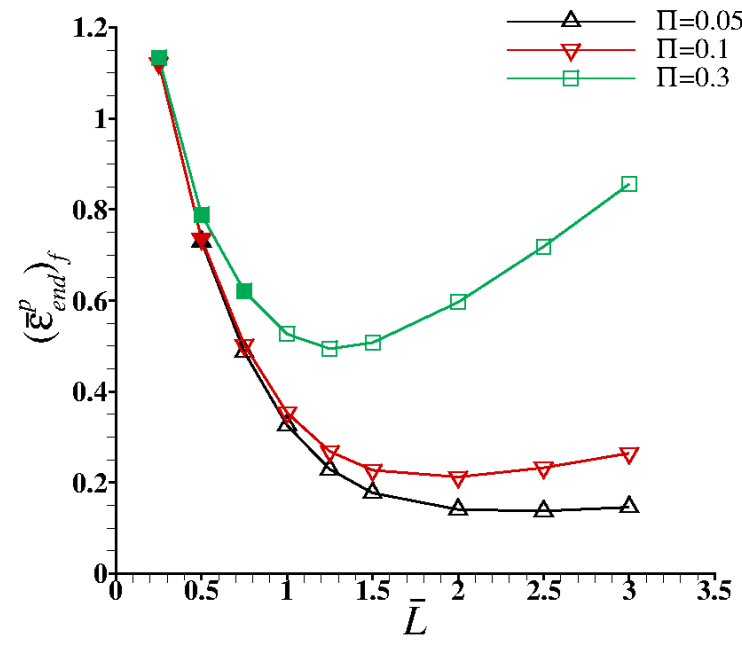

(b)

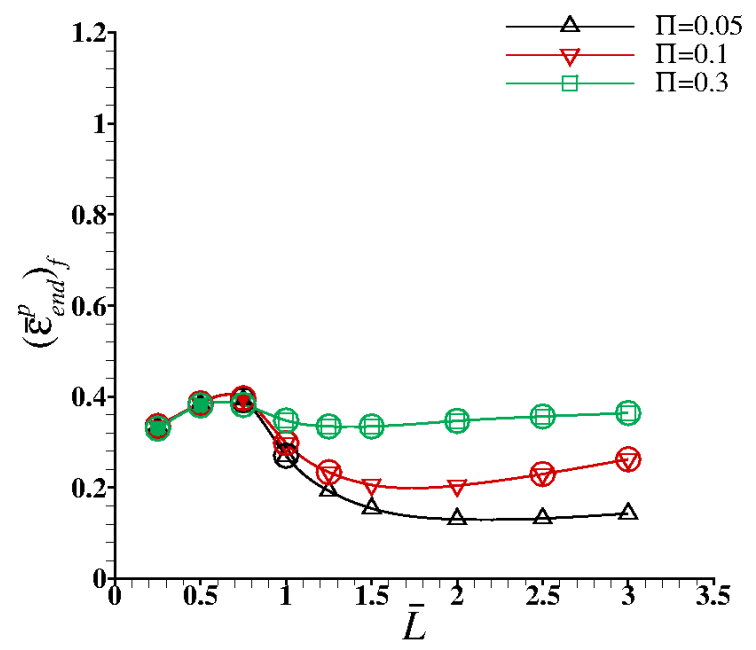

(d)

Fig. 6. Variation of the effective plastic strain at the end of the unit cell at failure, $\left(\bar{\varepsilon}_{\text {end }}^{p}\right)_{f}$, dictated either by the onset of localization or fracture in the center or at the surface of the mid-section of the unit cell (whichever occurs first), as a function of the normalized unit cell length, $\bar{L}$, for unit cells with initial porosity (a) $f_{0}=0$, (b) $f_{0}=0.01$, (c) $f_{0}=0.05$ and (d) $f_{0}=0.08$. The amplitude of the imperfection $A=0.002$ for all the unit cells. In the figure, open symbols correspond to the onset of localization in the center, closed symbols correspond to the onset of localization at the surface, encircled open symbols correspond to the onset of fracture in the center and encircled closed symbols correspond to the onset of fracture at the surface of the mid-section of the unit cell.

$\left(\bar{\varepsilon}_{\text {end }}^{p}\right)_{f}$ is either insensitive to the value of $\bar{L}$ or it decreases with decreasing value of $\bar{L}$. This is in contrast to the circumstances where necking precedes fracture as shown in Figs. 6(a) and (b). Also, in contrast to the circumstances where necking precedes fracture, for greater value of $\bar{L}$ when fracture precedes necking, the value of $\left(\bar{\varepsilon}_{\text {end }}^{p}\right)_{f}$ becomes less sensitive to both the values of $\bar{L}$ and imposed $\Pi$. So that for sufficiently high initial porosity levels and greater values of imposed $\Pi$ a critical value of $\bar{L}$ i.e. $\bar{L}_{c}$ does not exist.

The onset of fracture as modeled here, only depends on the evolution of porosity, which in turn predomi- nantly depends on the accumulated plastic strain for a fixed state of stress. This explains the decrease in the dependence of $\left(\bar{\varepsilon}_{\text {end }}^{p}\right)_{f}$ on the values of $\bar{L}$ and $\Pi$, for greater values of $\bar{L}$ and $\Pi$, when fracture precedes necking, Fig. 6(d). On the other hand, the decrease in the value of $\left(\bar{\varepsilon}_{\text {end }}^{p}\right)_{f}$ for smaller value of $\bar{L}$ can be explained with the results presented in Fig. 7. In this plot, we show the evolution of effective plastic strain, $\bar{\varepsilon}_{m i d}^{p}$, and hydrostatic stress, $\sigma_{h}$ in the mid-section of the unit cells with $\bar{\varepsilon}_{\text {end }}^{p}$, in two unit cells, $\bar{L}=0.25$ and 0.5 , with $f_{0}=0.08$ and subjected to $\Pi=0.1$. As shown in the figure, both the values of $\bar{\varepsilon}_{m i d}^{p}$, Fig. $7(\mathrm{a})$, and $\sigma_{h}$, 


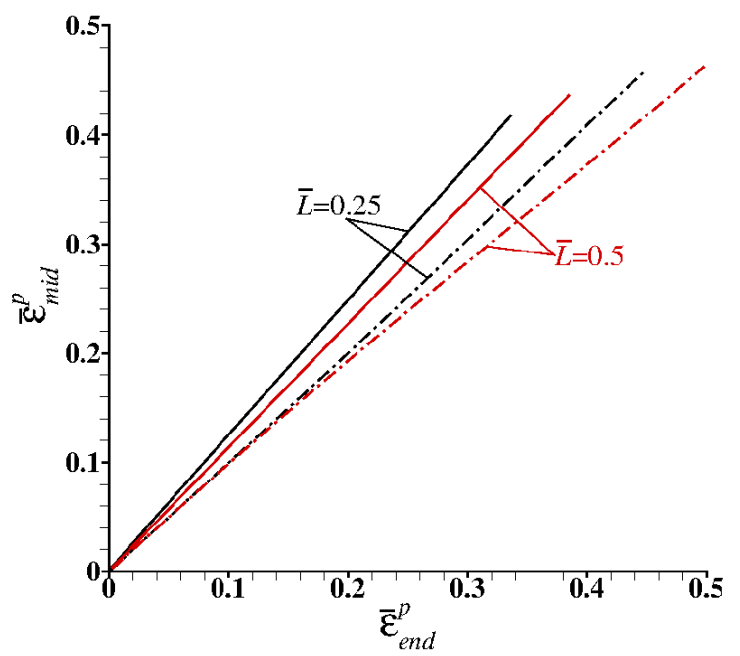

(a)

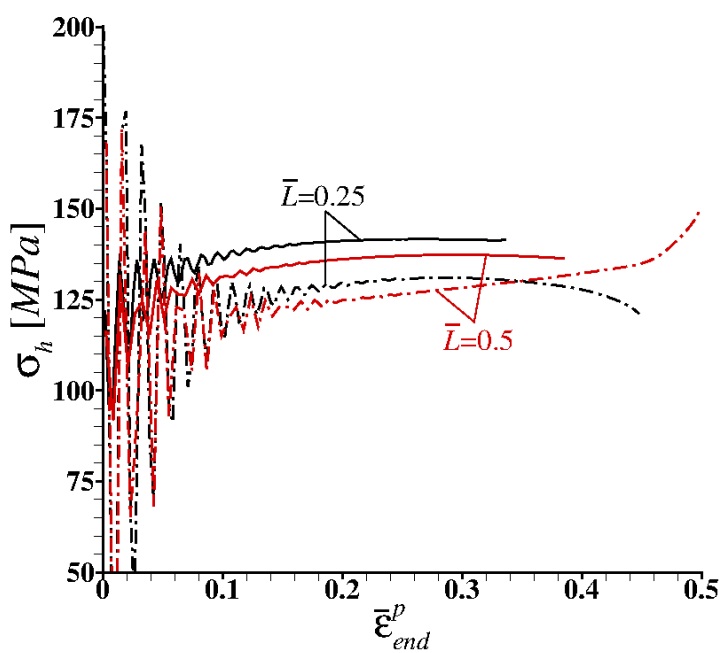

(b)

Fig. 7. Evolution of the (a) effective plastic strain, $\bar{\varepsilon}_{m i d}^{p}$, and (b) hydrostatic stress, $\sigma_{h}$ in the mid-section of the unit cell as a function of the effective plastic strain at the end of the unit cell, $\bar{\varepsilon}_{\text {end }}^{p}$ in two unit cells with normalized cell lengths $\bar{L}=0.25$ and 0.5 . For both the unit cells the imposed inertia parameter $\Pi=0.1$, initial porosity $f_{0}=0.08$ and the amplitude of the imperfection $A=0.002$. The dash-dot lines show the evolution of $\bar{\varepsilon}_{m i d}^{p}$ and $\sigma_{h}$ as a function of $\bar{\varepsilon}_{e n d}^{p}$ in the center while the solid lines show the same at the free surface of the unit cell.

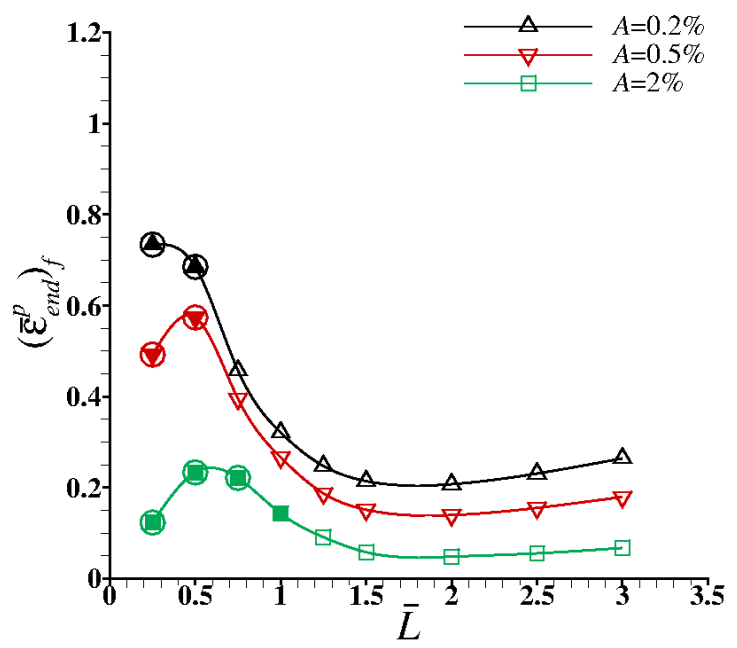

(a)

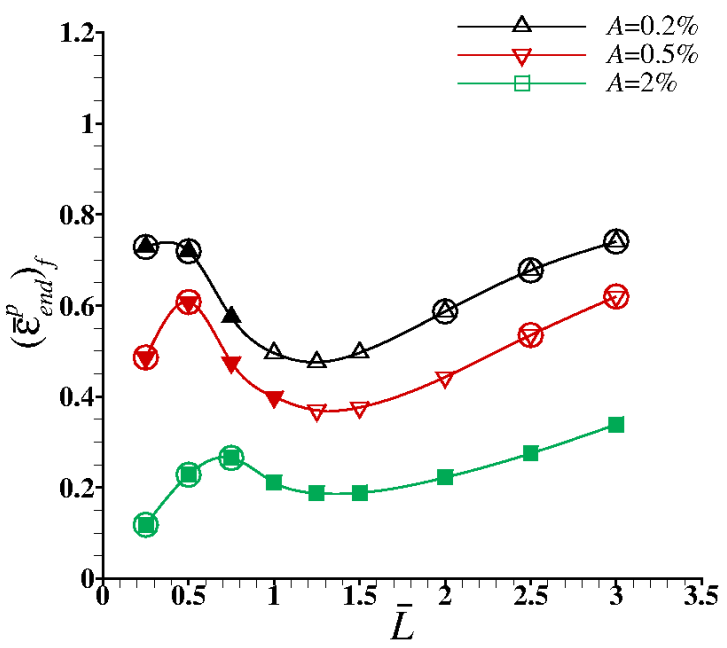

(b)

Fig. 8. Variation of the effective plastic strain at the end of the unit cell at failure, $\left(\bar{\varepsilon}_{\text {end }}^{p}\right)_{f}$, dictated by the onset of localization or fracture in the center or at the surface of the mid-section of the unit cell (whichever occurs first), as a function of the normalized cell length, $\bar{L}$, for unit cells subjected to inertia parameter (a) $\Pi=0.1$ and (b) $\Pi=0.3$. The initial porosity $f_{0}=0.05$ in all the unit cells. In the figure, open symbols correspond to the onset of localization in the center, closed symbols correspond to the onset of localization at the surface, encircled open symbols correspond to the onset of fracture in the center and encircled closed symbols correspond to the onset of fracture at the surface of the mid-section of the unit cell.

Fig. 7(b), in the mid-section of the unit cell increase as the value of $\bar{L}$ decreases, favoring the growth of porosity and promoting early fracture.

Next, we analyze the influence of the imperfection amplitude, $A$, on the predictions of the unit cell finite element calculations. The variation of $\left(\bar{\varepsilon}_{\text {end }}^{p}\right)_{f}$ with $\bar{L}$ for unit cells with initial porosity level $f_{0}=0.05$ for three values of $A$ and an imposed $\Pi=0.1$ are shown in Fig. 8(a). As shown in the figure, an increase in the value of $A$ results in a decrease in the value of $\left(\bar{\varepsilon}_{\text {end }}^{p}\right)_{f}$ for all the values of $\bar{L}$. The decrease in the value of $\left(\bar{\varepsilon}_{\text {end }}^{p}\right)_{f}$ with increasing value of $A$ is particularly signif- 
icant for smaller values of $\bar{L}$. For smaller values of $\bar{L}$, the range of the values of $\bar{L}$ for which fracture precedes necking increases with increasing value of $A$. For example, for $A=0.002$ fracture precedes necking for $\bar{L} \leq 0.5$ while for $A=0.02$ fracture precedes necking for $\bar{L} \leq 0.75$. Fig. 8(b) shows the variation of $\left(\bar{\varepsilon}_{\text {end }}^{p}\right)_{f}$ with $\bar{L}$ for unit cells with $f_{0}=0.05$ for three values of $A$ and an imposed $\Pi=0.3$. An increase in the value of imposed $\Pi$ delays the onset of localization, especially, for greater values of $\bar{L}$, thus, favoring the onset fracture before the onset of necking. For greater values of $\bar{L}$, the range of the values of $\bar{L}$ for which fracture precedes necking decreases with increasing value of $A$. This is contrary to the effect of $A$ on the range of $\bar{L}$ undergoing fracture for smaller values of $\bar{L}$. We note that an increase in the value of $A$ results in a slight increase in the value of the critical cell size, $\bar{L}_{c}$, for both the values of imposed $\Pi$. However, the effect of $A$ on the values of $\bar{L}_{c}$ and $\left(\bar{\varepsilon}_{\text {end }}^{p}\right)_{f}$ corresponding to $\bar{L}_{c}$ decreases with decreasing value of $A$.

\subsection{Comparison between finite element calcu- lations and linear stability analysis}

In this section, we compare the predictions of the unit cell finite element calculations and the linear stability analysis. For this we first need to define a criterion for the perturbation mode to turn into a necking mode (e.g. see refs. $[20,40])$. To this end, we follow the concept of effective instability introduced in ref. [45], which assumes that necking is triggered when the cumulative instability index $I$ reaches a value $I^{\text {neck }}$. The value of $I^{\text {neck }}$ can be determined from the results of the finite element calculations as shown in ref. [46]. In ref. [46], it was proposed that $I^{\text {neck }}$ corresponds to the critical cumulative instability index, $I_{c}$, see Section 4 , obtained from the linear stability analysis performed for the necking strain corresponding to $\bar{L}_{c}$ obtained from the finite element calculations. Here we rely on the finite element results for fully dense $\left(f_{0}=0\right)$ unit cells subjected to $\Pi=0.1$, Fig. 6 (a), to determine the value of $I^{\text {neck }}$. For fully dense material subjected to $\Pi=0.1$, the unit cell finite element calculations show that the value of critical normalized cell length $\bar{L}_{c}=2$, and the corresponding value of necking strain $\left(\bar{\varepsilon}_{\text {end }}^{p}\right)_{f}=0.214$. Next, using this necking strain as the strain for which the critical cumulative index is calculated in the linear stability analysis, the value of $I^{\text {neck }}$ is estimated to be 1 . We assume that the value of $I^{\text {neck }}$ is same for all perturbation wavelengths $(\bar{L})$, imposed inertia parameters $(\Pi)$, and initial porosity levels $\left(f_{0}\right)$. This is a rather strong assumption, as discussed later, but it enables us to calibrate the linear stability analysis in a simple manner, and check its predictive capabilities.

The variation of $\left(\bar{\varepsilon}_{e n d}^{p}\right)_{f}$ with $\bar{L}$ predicted using the unit cell finite element calculations, and the variation of the strain for which the condition $I^{\text {neck }}=1$ is met with the perturbation wavelength, $\bar{L}$, predicted using the linear stability analysis are compared in Fig 9 . The comparison in Fig 9 is carried out for initial porosity levels $f_{0}=0,0.05$ and 0.08 , and imposed inertia parameters $\Pi=0.05$ and 0.3. As shown in Fig 9(a), for $\Pi=0.05$ and $f_{0}=0$, there is a good qualitative and quantitative agreement between the predictions of the linear stability analysis and the unit cell finite element calculations for all the values of $\bar{L}$. However, for greater values of $f_{0}$ and smaller values of $\bar{L}$, the linear stability analysis over predicts the value of $\left(\bar{\varepsilon}_{e n d}^{p}\right)_{f}$. This is because for high initial porosity levels the unit cells with smaller values of $\bar{L}$ undergo fracture before necking which is more sensitive to the complex state of stress that develops in the three-dimensional unit cells (recall from Section 4 that the linear stability analysis models small perturbations of the fundamental one-dimensional solution). There is also a good qualitative agreement between the predictions of the linear stability analysis and unit cell finite element calculations for $\Pi=0.3$ and $f_{0}=0$, Fig 9(b). The linear stability analysis, however, under predicts the values of $\left(\bar{\varepsilon}_{\text {end }}^{p}\right)_{f}$ for greater values of $\bar{L}$. This suggests that the value of $I^{\text {neck }}$ is not constant and it depends on both the wavelength $\bar{L}$ and the value of imposed $\Pi$ (a detailed analysis of the dependence of $I^{\text {neck }}$ on the wavelength and inertia is left for a future work). Also, similar to $\Pi=0.05$ (Fig 9 (a)), for $\Pi=0.3$ (Fig 9(b)) as well, the linear stability analysis over predicts the value of $\left(\bar{\varepsilon}_{e n d}^{p}\right)_{f}$ for greater values of $f_{0}$ where the unit cells undergo fracture before necking.

In Fig. 10, we compare the variation in the values of $\bar{L}_{c}$ and $\bar{\varepsilon}_{c}$ with imposed $\Pi$ predicted using the unit cell finite element calculations and the linear stability analysis. For the finite element results, the value of $\bar{L}_{c}$ is the critical cell size and the value of $\bar{\varepsilon}_{c}$ is the value of $\left(\bar{\varepsilon}_{e n d}^{p}\right)_{f}$ corresponding to $\bar{L}_{c}$. While, for the linear stability analysis, the value of $\bar{L}_{c}$ is the critical wavelength and the value of $\bar{\varepsilon}_{c}$ is the critical strain (i.e. the minimum strain in the $\varepsilon-\bar{L}$ curves). The comparisons in Fig 10 are shown for initial porosity levels $f_{0}=0$ and 0.08. For all the cases, predictions of the linear stability analysis are obtained using two values of the cumulative instability index at necking, $I^{\text {neck }}=1$ and 6.72. The value of $I^{\text {neck }}=6.72$ was obtained in ref. [21] by calibrating $I^{\text {neck }}$ to the finite element results of multiple necking in a long cylindrical bar, free of geometric imperfections, and subjected to initial and boundary conditions consistent with dynamically expanding rings. The geometric imperfection included in the unit cell calculations carried out in this work results in a decrease in the value of $I^{\text {neck }}$ from 6.72 to 1 (this suggests that $I^{\text {neck }}$ also depends on the amplitude of the geometric imperfection, see ref. [46]).

As shown in Fig. 10, both, the unit cell finite element calculations and the linear stability analysis predict that the value of $\bar{L}_{c}$ decreases and the value of $\bar{\varepsilon}_{c}$ increases with increasing value of imposed П. However, the dependence of the value of $\bar{L}_{c}$ on the value of imposed $\Pi$ decreases for greater values of $\Pi$, in agreement 


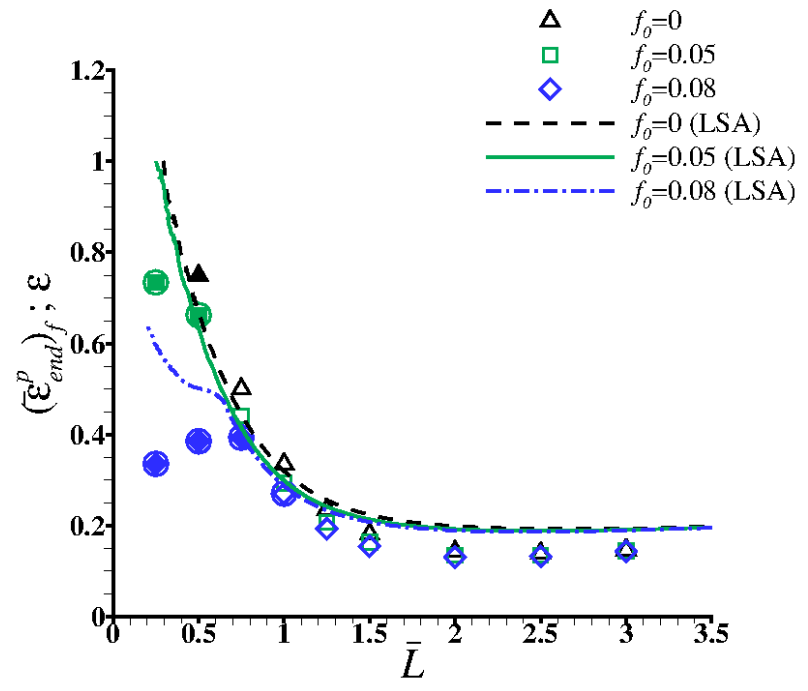

(a)

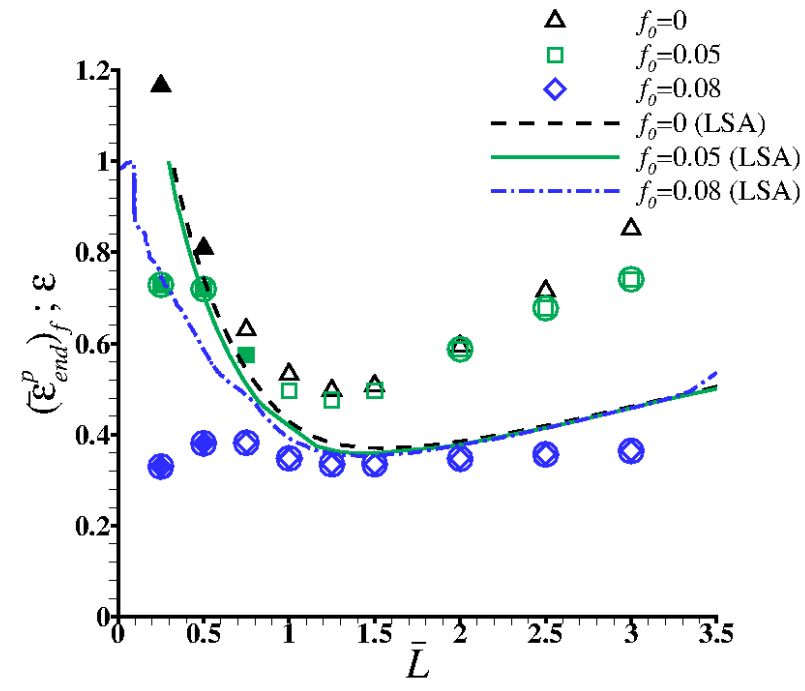

(b)

Fig. 9. Comparison between finite element and linear stability analysis (LSA) results. The finite element results show the variation of the effective plastic strain at the end of the unit cell at failure, $\left(\bar{\varepsilon}_{\text {end }}^{p}\right)_{f}$, dictated by the onset of localization or fracture in the center or at the surface of the mid-section of the unit cell (whichever occurs first), as a function of the normalized cell length, $\bar{L}$. The linear stability analysis results show the variation of the strain for which the condition $I^{\text {neck }}=1$ is met as a function of the perturbation wavelength $\bar{L}$. Results are shown for two different values of the inertia parameter: (a) $\Pi=0.05$ and (b) $\Pi=0.3$. The amplitude of the imperfection $A=0.002$ for all the unit cells and the value of the cumulative instability index at necking $I^{\text {neck }}=1$ for all perturbation wavelengths. The symbols have the same meaning as in Figs. 2, 4, 6 and 8.

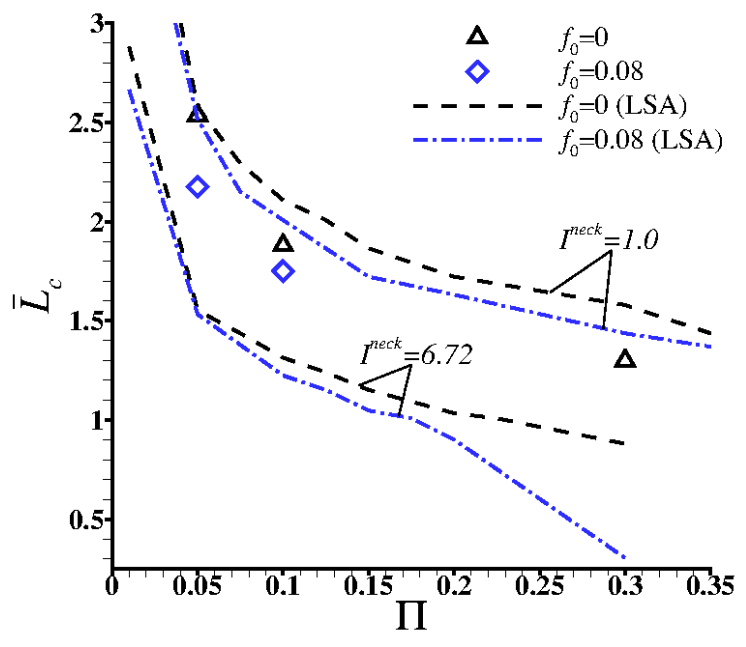

(a)

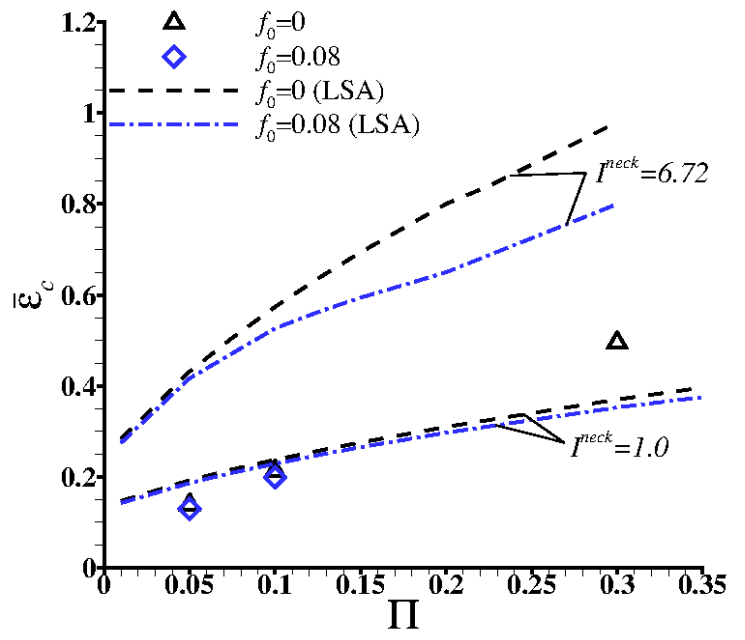

(b)

Fig. 10. Comparison between the variation of (a) critical cell size/wavelength, $\bar{L}_{c}$, and (b) critical necking/perturbation strain, $\bar{\varepsilon}_{c}$, with the imposed inertia parameter, $\Pi$, as predicted by the unit cell finite element calculations and the linear stability analysis (LSA). In the finite element calculations the amplitude of the imperfection $A=0.002$. The predictions of the linear stability analysis are obtained using the cumulative instability index at necking $I^{\text {neck }}=1$ and 6.72 .

with the finite element results pertaining to multiple necking in a ring or a long cylindrical bar in refs. [17,21]. Both, the unit cell finite element calculations and the linear stability analysis also predict slightly smaller values of $\bar{L}_{c}$ and $\bar{\varepsilon}_{c}$ for $f_{0}=0.08$ than for $f_{0}=0$. This suggests that increasing the initial porosity level in the material decreases (or increases) the average neck spacing (the number of necks) and the localization strain in multiple necking patterns. The linear stability analysis predictions of $\bar{L}_{c}$ and $\bar{\varepsilon}_{c}$ using $I^{\text {neck }}=1$ are in general good quantitative agreement with the finite element results whereas linear stability analysis using $I^{\text {neck }}=6.72$ 
under predicts the value of $\bar{L}_{c}$ and over predicts the value of $\bar{\varepsilon}_{c}$. This is consistent with the finite element results in Fig. 7 which show that an increase in the imperfection amplitude results in an increase in the value of $\bar{L}_{c}$ and a decrease in the value of $\left(\bar{\varepsilon}_{e n d}^{p}\right)_{f}$.

\section{Conclusions}

In this work, we have unraveled the complex interaction of initial porosity and inertia on the onset of necking and fracture in ductile materials subjected to dynamic loading conditions consistent with expanding rings. To this end, we have carried out a series of three-dimensional finite element calculations of unit cells with sinusoidal geometric perturbations. In the calculations, the material is modeled using a constitutive framework that includes many of the hardening and softening mechanisms that are characteristics of ductile metallic materials, such as strain hardening, strain rate hardening, thermal softening and damage-induced softening. The contribution of the inertia effect to the loading process is evaluated through a dimensionless inertia parameter that combines the effects of loading rate, material properties and cell size. The calculations are carried out for varying levels of initial porosity and a wide range of the value of imposed inertia parameter. From all the unit cell finite element calculations, strain to failure dictated by necking and/or fracture are measured. The finite element results are also compared with the predictions of linear stability analysis of necking instabilities in porous ductile materials. The key conclusions of this work are as follows:

- Low initial porosity levels favor the onset of necking before fracture, and high initial porosity levels favor the onset of fracture before necking, especially, at high loading rates where inertia effects delay the onset of necking.

- For the levels of initial porosity and imposed inertia parameter where the onset of necking precedes fracture, a critical unit cell size (cell size with minimum necking strain) as a function of initial porosity and imposed inertia parameter can be identified.

- For the levels of initial porosity and imposed inertia parameter where the onset of necking precedes fracture, the critical unit cell size obtained from finite element calculations and the critical wavelength for necking obtained from linear stability analysis decreases with increasing initial porosity levels and the value of the inertia parameter.

- For sufficiently high initial porosity levels and imposed inertia parameter where the onset of fracture precedes necking, the value of strain at failure obtained from finite element calculations is found to be less sensitive to the size of the unit cell. So that it is not possible to identify the critical cell size.

- The linear stability analysis, despite several simplifying assumptions, shows a very good correlation with the predictions of unit cell finite element calculations under the circumstances where the onset of necking precedes fracture.

The most important message of this paper is that when necking precedes fracture, for fully dense materials or materials with low initial porosity, there exists a critical cell size or a critical wavelength for which the deformation energy required to trigger a neck is minimum. This critical cell size or critical wavelength likely represents the average neck spacing in the multiple necking pattern of dynamically expanding rings. However, for materials with high initial porosity, an increase in inertial loading may favor early fracture, precluding the development of a necking pattern. Under these circumstances, when fracture precedes necking, no critical cell size or critical wavelength dictates the fragmentation of dynamically expanding ring.

\section{Acknowledgements}

The finite element calculations reported on were carried out using high performance research computing resources provided by Texas A\&M University. J.A.R.M. acknowledges the financial support provided by the European Research Council under the European Union's Horizon 2020 research and innovation programme (Project PURPOSE, grant agreement 758056).

\section{References}

[1] Niordson, F. L., 1965. "A unit for testing materials at high strain rates". Experimental Mechanics, 5, pp. 29-32.

[2] Grady, D. E., and Benson, D. A., 1983. "Fragmentation of metal rings by electromagnetic loading". Experimental Mechanics, 12, pp. 393-400.

[3] Grady, D. E., Kipp, M. E., and Benson, D. A., 1984. "Energy and statistical effects in the dynamic fragmentation of metal rings". In Mechanical properties at high strain rates, J. Harding, ed., , Institute of Physics, pp. 315-320.

[4] Altynova, M., Hu, X., and Daehn, G. S., 1996. "Increased ductility in high velocity electromagnetic ring expansion". Metallurgical Transactions A, 27, pp. $1837-1844$.

[5] Pandolfi, A., Krysl, P., and Ortiz, M., 1999. "Finite element simulation of ring expansion and fragmentation: The capturing of length and time scales through cohesive models of fracture". International Journal of Fracture, 95, pp. 297-297.

[6] Sørensen, N. J., and Freund, L. B., 2000. "Unstable neck formation in a ductile ring subjected to impulsive radial loading". International Journal of Solids and Structures, 37, pp. 2265-2283.

[7] Becker, R., 2002. "Ring fragmentation predictions using the Gurson model with material stability con- 
ditions as failure criterion". International Journal of Solids and Structures, 39, pp. 3555-3580.

[8] Mercier, S., and Molinari, A., 2004. "Analysis of multiple necking in rings under rapid radial expansion". International Journal of Impact Engineering, 30, pp. 403-419.

[9] Triantafyllidis, N., and Waldenmyer, J. R., 2004. "Onset of necking in electro-magnetically formed rings". Journal of the Mechanics and Physics of Solids, 52, pp. 2127-2148.

[10] Zhang, H., and Ravi-Chandar, K., 2006. "On the dynamics of necking and fragmentation - I. Realtime and post-mortem observations in $\mathrm{Al} 6061-\mathrm{O}$ ". International Journal of Fracture, 142, pp. 183217.

[11] Zhou, F., Molinari, J. F., and Ramesh, K. T., 2006. "An elasto-visco-plastic analysis of ductile expanding ring". International Journal of Impact Engineering, 33, pp. 880-891.

[12] Grady, D., 2006. Fragmentation of rings and shells the legacy of N. F. Mott, 1 ed. Springer-Verlag Berlin Heidelberg.

[13] Rusinek, A., and Zaera, R., 2007. "Finite element simulation of steel ring fragmentation under radial expansion". International Journal of Impact Engineering, 34, pp. 799-822.

[14] Zhang, H., and Ravi-Chandar, K., 2008. "On the dynamics of necking and fragmentation - II. Effect of material properties geometrical constraints and absolute size". International Journal of Fracture, 150, pp. 3-36.

[15] Zhang, H., and Ravi-Chandar, K., 2010. "On the dynamics of localization and fragmentation-IV. Expansion of Al 6061-O tubes". International Journal of Fracture, 163, pp. 41-65.

[16] Janiszewski, J., 2012. "Ductility of selected metals under electromagnetic ring test loading conditions". International Journal of Solids and Structures, 49, pp. 1001-1008.

[17] Rodríguez-Martínez, J. A., Vadillo, G., FernándezSáez, J., and Molinari, A., 2013. "Identification of the critical wavelength responsible for the fragmentation of ductile rings expanding at very high strain rates". Journal of the Mechanics and Physics of Solids, 61, pp. 1357-1376.

[18] Cliche, N., and Ravi-Chandar, K., 2018. "Dynamic strain localization in magnesium alloy AZ31B-O". Mechanics of Materials, 116, pp. 189-201.

[19] Hu, X., and Daehn, G. S., 1996. "Effect of velocity on flow localization in tension". Acta Materialia, 44, pp. 1021-1033.

[20] Guduru, P. R., and Freund, L. B., 2002. "The dynamics of multiple neck formation and fragmentation in high rate extension of ductile materials". International Journal of Solids and Structures, 39, pp. $5615-5632$.

[21] N'souglo, K. E., Srivastava, A., Osovski, S., and Rodríguez-Martínez, J. A., 2018. "Random distri- butions of initial porosity trigger regular necking patterns at high strain rates". Proceedings of the Royal Society A: Mathematical, Physical and Engineering Sciences, 474, p. 20170575.

[22] Han, J. B., and Tvergaard, V., 1995. "Effect of inertia on the necking behaviour of ring specimens under rapid axial expansion". European Journal of Mechanics A/Solids, 14, pp. 287-307.

[23] ABAQUS/Explicit, 2010. Abaqus Explicit v6.10 User's Manual, version 6.10 ed. ABAQUS Inc., Richmond, USA.

[24] Xue, Z., Vaziri, A., and Hutchinson, J., 2008. "Material aspects of dynamic neck retardation". Journal of the Mechanics and Physics of Solids, 56, pp. 93113.

[25] Gurson, A., 1977. "Continuum theory of ductile rupture by void nucleation and growth. Part I: Yield criteria and flow rules for porous ductile media". ASME Journal of Engineering Materials and Technology, 99, pp. 2-15.

[26] Tvergaard, V., and Needleman, A., 1984. "Analysis of the cup-cone fracture in a round tensile bar". Acta Metallurgica, 32, pp. 157-169.

[27] Tvergaard, V., 1981. "Influence of voids on shear band instabilities under plane strain conditions". International Journal of Fracture, 17(4), pp. 389407.

[28] Tvergaard, V., 1982. "On localization in ductile materials containing spherical voids". International Journal of Fracture, 18(4), pp. 237-252.

[29] Pan, J., Saje, M., and Needleman, A., 1983. "Localization of deformation in rate sensitive porous plastic solids". International Journal of Fracture, 21(4), pp. 261-278.

[30] Srivastava, A., Ponson, L., Osovski, S., Bouchaud, E., Tvergaard, V., and Needleman, A., 2014. "Effect of inclusion density on ductile fracture toughness and roughness". Journal of the Mechanics and Physics of Solids, 63, pp. 62-79.

[31] Srivastava, A., Osovski, S., and Needleman, A., 2017. "Engineering the crack path by controlling the microstructure". Journal of the Mechanics and Physics of Solids, 100, pp. 1-20.

[32] Osovski, S., Srivastava, A., Ponson, L., Bouchaud, E., Tvergaard, V., Ravi-Chandar, K., and Needleman, A., 2015. "The effect of loading rate on ductile fracture toughness and fracture surface roughness". Journal of the Mechanics and Physics of Solids, 76, pp. 20-46.

[33] Osovski, S., Srivastava, A., Williams, J., and Needleman, A., 2015. "Grain boundary crack growth in metastable titanium $\beta$ alloys". Acta $M a-$ terialia, 82, pp. 167-178.

[34] Belytschko, T., Chiapetta, R. L., and Bartel, H. D., 1976. "Efficient large scale non-linear transient analysis by finite elements". International Journal for Numerical Methods in Engineering, 10(3), pp. 579-596. 
[35] Peirce, D., Shih, C. F., and Needleman, A., 1984. "A tangent modulus method for rate dependent solids". Computers \& Structures, 18(5), pp. 875887.

[36] Tvergaard, V., 1982. "Influence of void nucleation on ductile shear fracture at a free surface". Journal of the Mechanics and Physics of Solids, 30(6), pp. 399-425.

[37] Fressengeas, C., and Molinari, A., 1985. "Inertia and thermal effects on the localization of plastic flow". Acta Metallurgica, 33, pp. 387-396.

[38] Fressengeas, C., and Molinari, A., 1994. "Fragmentation of rapidly stretching sheets". European Journal of Mechanics A/Solids, 13, pp. 251-268.

[39] Mercier, S., and Molinari, A., 2003. "Predictions of bifurcations and instabilities during dynamic extensions". International Journal of Solids and Structures, 40, pp. 1995-2016.

[40] Mercier, S., Granier, N., Molinari, A., Llorca, F., and Buy, F., 2010. "Multiple necking during the dynamic expansion of hemispherical metallic shells, from experiments to modelling". Journal of the Mechanics and Physics of Solids, 58, pp. 955-982.

[41] Godinger, A., Rotbaum, Y., Vaz-Romero, A., Rodríguez-Martínez, J. A., and Rittel, D., 2017. "On the relation between shape imperfections of a specimen and necking growth rate under dynamic conditions". International Journal of Engineering Science, 119, pp. 278-287.

[42] El Maï, S., Mercier, S., Petit, J., and Molinari, A., 2014. "An extension of the linear stability analysis for the prediction of multiple necking during dynamic extension of round bar". International Journal of Solids and Structures, 51, pp. 3491-3507.

[43] Vaz-Romero, A., Rodríguez-Martínez, J. A., Mercier, S., and Molinari, A., 2017. "Multiple necking pattern in nonlinear elastic bars subjected to dynamic stretching: The role of defects and inertia". International Journal of Solids and Structures, 125, pp. 232-243.

[44] Knoche, P., and Needleman, A., 1993. "The effect of size on the ductility of dynamically loaded tensile bars". European Journal of Mechanics A/Solids, 12, pp. 585-601.

[45] Dudzinski, D., and Molinari, A., 1991. "Perturbation analysis of thermoviscoplastic instabilities in biaxial loading". International Journal of Solids and Structures, 27, pp. 601-628.

[46] N'souglo, K. E., Rodríguez-Martínez, J. A., and Cazacu, O., 2019. "The effect of plastic dissipation in the formation of dynamic necking instabilities in incompressible materials displaying tensioncompression asymmetry". Submitted for publication. 